\title{
CORRESPONDENCE
}

\section{Which is the principal early-life infection-related risk factor for asthma?}

\section{To the Editors:}

In a previous issue of the European Respiratory Journal, Kusel et al. [1] reported very interesting 10-yr results of their prospective birth cohort study. Originally, 263 children at risk for asthma and allergy were recognised pre-natally and recruited as newborns, and $198(75.3 \%)$ attended the 5-yr [2] and 147 (55.9\%) the 10-yr follow-up study [1]. The drop-out rate of $44 \%$ is understandable but needs evaluation. The data were collected very carefully during the first year including diaries completed by parents and home visits with sampling for virus detection by PCR during all acute infections [2]. Respiratory infections were classified as upper (URI) and lower (LRI), and both URIs and LRIs further as wheezy and nonwheezy.

At age 10 yrs, $18.4 \%$ of the 147 children had current asthma and $20.4 \%$ persistent wheeze [1]. During the first year of life, $35.8 \%$ had experienced at least one LRI with fever and/or wheeze. The figure was $26.5 \%$ for febrile LRI and, likewise, $26.5 \%$ for wheezy LRI. Atopy determined by skin-prick tests to a panel of two food and five inhaled allergens was present in $31(21.1 \%)$ infants at age 6 months, and in $63(42.9 \%)$ and $56(38.1 \%)$ children at age 5 and 10 yrs, respectively.

In adjusted analyses, children who had wheezy or febrile LRI at age $<12$ months and were atopic by age 2 yrs were at increased risk for asthma or persistent wheeze at 10 yrs [1]. Wheezy LRI associated with human rhinovirus (HRV) was a risk factor for asthma and persistent wheeze only in children with atopy onset after 2 yrs. However, wheezy LRI associated with respiratory syncytial virus (RSV) was not a risk factor in either group formed by atopy onset [1].

The authors highlight the waning of the association between wheezy LRI at age $<12$ months and asthma or persistent wheezy ascertained at 10 versus 5 yrs [1]. Instead, they stress the role of the severity of early-life respiratory infection, which in their study was estimated by fever and LRI versus URI.

We have prospectively followed up, over 25 yrs, 83 children who were hospitalised for bronchiolitis at age $<24$ months with chest radiographs taken on admission in all cases. An interstitial infiltration was found in $46(57 \%)$ children. Follow-up studies were performed at 4.5-6.0, 8.5-10.0, 14.0-16.0, 18.5-20.0 and 26$28 \mathrm{yrs}$, and we found no evidence for an association between current asthma at any time and the severity of bronchiolitis, assessed by radiological infiltration [3]. In addition, asthma was consistently more common in these two wheezing groups than in the 44 children hospitalised for pneumonia alone.

The multivariate analyses used by KuseL et al. [1] were adjusted for sex, breastfeeding, pet ownership, older siblings, tobacco smoke exposure and daycare attendance. Atopy at age 6 months was not, but atopy at age 2 and / or 5 yrs was, a risk factor for both asthma and persistent wheeze at 10 yrs. Atopy was taken into account in the analyses by performing stratified analyses in relation to atopy onset by and after 2 yrs.

However, this modelling does not answer the question of whether the severity (fever in the present study) of respiratory infection in infancy is a risk factor for later asthma or persistent wheezing independently of the associated virus or the presence of wheeze. In addition, there might be certain time windows for the virus-specific or nonspecific influences of infections to airways or to immunity even within the first 12 months of life. Perhaps such modelling would be worth trying here, and grouping might be realistic. Only $9.3 \%$ of infants with LRI had both wheeze and fever. Febrile LRI did not recur in any child and wheezy LRI recurred in only 14 children. No data were presented on fever and wheeze URIs, which may, of course, be both confounding and disease-modifying factors.

In our post-bronchiolitis studies, HRV bronchiolitis (or non-RSV bronchiolitis in older studies) increased the later asthma risk compared with RSV bronchiolitis, with some waning of the effects of both viruses from $7 \mathrm{yrs}$ of age onwards [4], in line with the waning between 5 to $10 \mathrm{yrs}$ in the present study [1]. In addition, RSV and HRV seem to have different effects on lung function presenting at least at 7 and $12 \mathrm{yrs}$ of age. RSV bronchiolitis was associated with a more restrictive and HRV with a more obstructive pattern of subnormal of lung function [5].

As the authors summarised, severe LRI in infancy is a major risk factor for asthma and persistent wheeze at age $10 \mathrm{yrs}$ in high-risk children [1]. However, the specific roles of different viruses in early life need further attention, as does the question of whether the severity/fever is an independently significant risk factor. Lung function results, which hopefully have been obtained and will be reported in the near future, may shed more light on both open questions.

Matti Korppi ${ }^{*}$, Mari Hyvärinen ${ }^{\#}$ and Eija Piippo-Savolainen ${ }^{\#}$ *Paediatric Research Center, Tampere University and University Hospital, Tampere, and "Dept of Paediatrics, Kuopio University Hospital, Kuopio, Finland.

Correspondence: M. Korppi, Paediatric Research Center, Tampere University Hospital, FIN-33140 Tampere, Finland. E-mail: matti.korppi@uta.fi

Statement of Interest: None declared. 


\section{REFERENCES}

1 Kusel MMH, Kebadze T, Johnston SL, et al. Febrile respiratory illnesses in infancy and atopy are risk factors for persistent asthma and wheeze. Eur Respir J 2012; 39: 876-882.

2 Kusel MM, de Klerk NH, Kebadze T, et al. Early-life respiratory viral infections, atopic sensitization, and risk of subsequent development of persistent asthma. J Allergy Clin Immunol 2007; 119: 1105-1110.

3 Piippo-Savolainen E, Ruotsalainen M, Korppi M. Long-term outcome after bronchiolitis: no association with the invasiveness of the infection. J Allergy Clin Immunol 2009; 124: 1121.
4 Hyvärinen MK, Kotaniemi-Syrjänen A, Reijonen TM, et al. Teenage asthma after severe early childhood wheezing: an 11-year prospective follow-up. Pediatr Pulmonol 2005; 40: 316-323.

5 Hyvärinen MK, Kotaniemi-Syrjänen A, Reijonen TM, et al. Lung function and bronchial hyper-responsiveness 11 years after hospitalization for bronchiolitis. Acta Paediatr 2007; 96: 1464-1469.

\section{Linezolid: safety and efficacy monitoring}

\section{To the Editors:}

We read with interest the recent European Respiratory Journal article by SINGLA et al. [1], which described the treatment outcome of 29 (pre-)extensively drug-resistant (XDR) tuberculosis (TB) patients from Delhi, India. All patients received linezolid as part of their anti-TB regimen. The high percentage of favourable treatment outcomes in the study led SinGLA et al. [1] to conclude that "Linezolid could have played a key role". However, in our opinion, conclusions on the role of a single agent, such as linezolid, are difficult to draw from a series of cases without controls, in which every patient received linezolid in addition to an injectable antimycobacterial agent and a fluoroquinolone. Indeed, the important role of later-generation fluoroquinolones is addressed, but neither drug sensitivity testing (DST) nor drug concentration monitoring for linezolid was performed. Therefore, linezolid treatment itself could even be subtherapeutic [2].

SINGLA et al. [1] conclude that "an aggressive, comprehensive management programme using linezolid along with other drugs can favourably treat significant number of patients". Although we concur with this statement, a closer look at the management programme applied in this study suggests that the programme might not be too aggressive or comprehensive. For instance, no directly observed therapy (DOT) was applied, nor did the patients receive nutritional or good psychosocial support. Compliance was only assessed indirectly by checking empty blister packs. Since noncompliance could lead to treatment failure and increase of resistance against the few drugs that are still effective in (pre)XDR-TB treatment, we would strongly advise to abandon DOT only in exceptional cases where compliance is highly probable [3]. Therapeutic drug monitoring (TDM) can be recommended to ensure adequate drug exposure during treatment. In rural areas, dried blood spot analysis may enable TDM by offering an affordable tool for drug concentration measurement in a centralised laboratory using stable, easy-to-obtain samples [4].

The very low incidence of major adverse events (AEs) of $10.3 \%$ reported by SINGLA et al. [1] is in contrast with findings in literature, where $41.2 \%$ of 85 multidrug-resistant/XDR-TB patients treated with linezolid experienced major AEs [5]. The authors provided no explanation for low AE incidence found in their study. Perhaps the fact that temporary discontinuation of linezolid was scored as a minor adverse event, along with the absence of DOT, resulted in a lower score of AEs in the study by SINGLA et al. [1]. Unfortunately, the authors provided no information on the manufacturer of the linezolid. Only the low cost of linezolid of less than US\$1 per tablet is mentioned, compared with approximately US\$80 per tablet in the Netherlands. It is well established that counterfeit drugs pose a great threat and counterfeit drugs sometimes contain little to none of the claimed drug [6]. Although there is no evidence that the administered drugs in this study were counterfeit, it also cannot be excluded based on the information provided by SINGLA et al. [1]. This, combined with the absence of DOT and TDM, could very well be a reason for the low incidence of major AEs as observed in the study by SINGLA et al. [1].

In our opinion, only a randomised controlled trial of linezolid versus a placebo, in addition to an adequate background regimen using DST and TDM, will provide comprehensive results on efficacy and safety of linezolid as potential drug for (pre-)XDR-TB treatment regimen.

\section{Mathieu S. Bolhuis, Arianna D. Pranger and Jan-Willem C. Alffenaar}

Dept of Hospital and Clinical Pharmacy, University Medical Center Groningen, University of Groningen, Groningen, The Netherlands.

Correspondence: M.S. Bolhuis, Dept of Hospital and Clinical Pharmacy, University Medical Center Groningen, University of Groningen, PO Box 30.001, 9700 RB Groningen, The Netherlands. E-mail: m.s.bolhuis@umcg.nl

Statement of Interest: A statement of interest for J-W.C. Alffenaar can be found at www.erj.ersjournals.com/site/ misc/statements.xhtml

\section{REFERENCES}

1 Singla R, Caminero JA, Jaiswal A, et al. Linezolid: an effective, safe and cheap drug for patients failing multidrug-resistant treatment in India. Eur Respir J 2012; 39: 956-962.

2 Alffenaar JW, Kosterink JG, van Altena R, et al. Limited sampling strategies for therapeutic drug monitoring of linezolid in MDR-TB patients. Ther Drug Monit 2010; 32: 97-101. 